\title{
Erratum: Effective medium theory for magnetodielectric composites: Beyond the long-wavelength limit [Phys. Rev. B 74, 085111 (2006)]
}

Ying Wu, Jensen Li, Zhao-Qing Zhang, and C. T. Chan

(Received 19 October 2015; published 4 November 2015)

DOI: 10.1103/PhysRevB.92.199901

PACS number(s): 41.20.Jb, 42.70.Qs, 78.20.Ci, 99.10.Cd

The original paper contains a typographical error in Eq. (6). The derivative sign (prime) on the Hankel function in the first term of the denominator is missing. The correct form of Eq. (6) is

$$
D_{m}=\frac{\mu_{0} k_{s} J_{m}^{\prime}\left(k_{s} r_{s}\right) J_{m}\left(k_{0} r_{s}\right)-\mu_{s} k_{0} J_{m}\left(k_{s} r_{s}\right) J_{m}^{\prime}\left(k_{0} r_{s}\right)}{\mu_{s} k_{0} J_{m}\left(k_{s} r_{s}\right) H_{m}^{\prime(1)}\left(k_{0} r_{s}\right)-\mu_{0} k_{s} J_{m}^{\prime}\left(k_{s} r_{s}\right) H_{m}^{(1)}\left(k_{0} r_{s}\right)}
$$

This error does not affect any results in the paper. 